\title{
Tracing Interaction in Distributed Collaborative Learning
}

\author{
Daniel Suthers and Richard Medina \\ Laboratory for Interactive Learning Technologies \\ Dept. of Information and Computer Sciences, University of Hawai $i$ at Manoa \\ 1680 East West Road, POST 309, Honolulu, HI 96822, USA \\ http://lilt.ics.hawaii.edu \\ collaborative-representations@hawaii.edu
}

\begin{abstract}
We seek to understand how learning phenomena are produced by entanglements and alignments of the activities of multiple individuals in technologymediated environments. How do participants construct new understandings in an online environment? How do communities of interest and practice emerge and sustain themselves in shared virtual spaces? And how do designed environments influence these processes through their affordances? To answer such questions, it is necessary to trace out activity that is distributed across time and space and media, following the trajectories of people, the transformation and spread of ideas, and the movement of artifacts: these are three ontological perspectives on one phenomenon. Multiple analytic challenges are identified in this paper, including the distributed nature of the data, the contingent nature of human behavior, understanding nonverbal behavior, selective attention to large data sets, and multi-scale phenomena. As one part of our solution, we have developed mediaindependent representations of contingencies between mediated actions and of trajectories of participation that intersect on persistent objects. This paper describes the contingency graph representation, gives an example of its use in analyzing the development of shared representational practices, and discusses further challenges. Important questions remain concerning the extent to which interactional accounts can remain productive as we grapple with larger data sets and emergent phenomena, and whether a productive interplay between interactional and aggregate accounts are possible that together inform design.
\end{abstract}

\section{Introduction}

Our prior research has generally been concerned with a fundamental two-sided question: how are the affordances of designed media appropriated for intersubjective meaning-making, and how can the availability and salience of these affordances influence meaning-making processes? This generalized research question has various instantiations in the settings that we study. The research program began with a series of laboratory studies of "representational guidance" in dyadic problem solving and learning that showed the influence of notation on certain categories of behavior (Suthers \& Hundhausen, 2003). Yet, these studies did not analyze how the participants collaborated with these notations. We therefore undertook analyses of interaction to understand issues such as how argumentation and problem solving can take place through joint synchronous manipulation of shared workspaces (Suthers, 2006a), how participants in an asynchronous setting not only share information but come to agreement on its interpretation (Suthers, Dwyer, Vatrapu \& Medina, 2007), and how representational practices are invented and develop in such environments (Dwyer \& Suthers, 2006; Medina \& Suthers, 2008). Although our 
data from these studies involved dyads only, we have begun to bring similar analytic questions to the interaction of larger (yet still relatively "small") groups that are described next.

During the same time, our laboratory was responsible for developing and supporting a statewide community of public school teachers through an online environment that included collaborative workspaces and shared resources (hnlc.org; see Suthers et al., 2004; Suthers, Yukawa, \& Harada, 2007), and we have applied a similar environment to a community-oriented approach to online graduate education (discourse.ics.hawaii.edu). Both of these efforts provide us with further data on small group processes of argumentation, problem solving, interpretation, and invention of practices. Additionally, they offer larger scale phenomena of interest. In both of these efforts, we sought to embed task-oriented groups (e.g., school teams and courses, respectively, as well as special interest groups) in common digital environments that support opportunistic formation of social relationships and resource sharing. The objective is to enable the formation of "transcendent communities" beyond the scope of the teams or courses that brought members to the environment in the first place (Joseph, Lid, \& Suthers, 2007). This objective leads to our interest in analyzing boundary spanning and other phenomena concerning how new social relationships form in online environments and their consequences.

Although diverse in terms of the settings and questions addressed, these studies share similar analytic challenges, some of which are addressed in this paper. In each case it is necessary to find the phenomena of interest in the trajectories and entanglements of the activities of multiple individuals - activity that is distributed across time, space and media. The remainder of this paper addresses the theoretical framework that motivates the research, the kinds of data needed to address the research questions, the greatest analytic challenges we are confronted with; how we have begun to address these challenges; the challenges that remain; and a summary of how addressing these analytic challenges advances our understanding of how people learn in technology-mediated environments. An example of analysis of dyadic problem solving is given, this being the application in which our approach is more completely worked out. Application to the analysis of larger groups and social networks is discussed in the section on future challenges.

\section{Theoretical Framework}

\section{What is the theoretical framework that motivates the research? ${ }^{1}$}

This research is concerned with collaborative and networked learning in technology-mediated environments. Our theoretical framework addresses the nature of "collaborative and networked learning" and "technology-mediated."

\subsection{Collaborative and Networked Learning}

We are generally interested in learning "in the context of joint activity" (Koschmann, 2002). This learning may occur at various social granularities and types of "joint activity," ranging from small numbers of tightly coupled collaborators who maintain a "joint conception of a problem" (Dillenbourg, 1999; Teasley \& Roschelle, 1993) or engage in "group cognition" (Stahl, 2006), to learning in "virtual communities" (Renninger \& Shumar, 2002) and in loosely associated networks of individuals (Castells, 2001; Jones, Dirckinck-Holmfeld, \& Lindstrom, 2006). There are several ways in which social contexts can be seen as contributing to learning, briefly reviewed in (Suthers, 2006b). We are particularly interested in processes in which individual

\footnotetext{
${ }^{1}$ This paper is organized according to questions posed to authors by the AERA symposium organizers.
} 
attempts at meaning-making influence others through the technological environment and lead to intersubjective meaning-making. To understand technology mediated collaborative and networked learning we need to trace out trajectories of intra- and inter-subjective meaningmaking and relate these to the media used.

Our approach to understanding individual and collective trajectories of meaning-making is influenced by ethnomethodology's program of identifying the methods by which "members" produce recognizable accomplishments (Garfinkel, 1967) such as learning (Koschmann et al., 2005), and is also influenced by actor-network theory's program of "reassembling the social" (Latour, 2005b) from a network of human and technological actors. But rather than showing how social order is constructed in interaction, our program is oriented towards the problem of designing for learning. "Ethnomethodological indifference" and "relevance" (Koschmann, Stahl, $\&$ Zemel, 2007) are tempered by our need to select events that are interesting from a learning perspective. The concept of "learning" is a judgment that something worthwhile has taken place in a particular episode. Our analysis works back from this recognition to trace out the interactional accomplishment of meaning-making that lead to that learning. We speak of "meaning-making" because we maintain that participants are continuously trying to make sense of their experience at multiple levels (e.g., concerning the task, interpersonal relations, and normative behavior; Bronckart, 1995). Participants are engaged in "doing" meaning-making; the question of learning is an evaluation of the consequences of that doing.

The basic unit of interaction for the analysis of intersubjective meaning-making is "uptake": the event of a participant taking up a prior act or reification of that act as having some relevance for ongoing participation (Suthers, 2006a; Suthers, Dwyer, Medina, \& Vatrapu, 2007a). Uptake includes but is more general than "transactivity" (Berkowitz \& Gibbs, 1979) in that uptake need not be directed towards a particular other actor, and indeed can occur in realms of participation in which the originating actor is not participating. We must infer uptake from observable contingencies between actions, which requires a separate representation of this evidence.

\subsection{Technology-Mediated}

We call actions taken by participants in the technological environment "media coordinations" because these actions are the means through which participants coordinate between personal and public realms (Hutchins, 1995). Other literature uses the term "contribution," but we desire a term that does not imply a conversational setting or a particular kind of participatory intention.

The technological medium is not neutral. A given medium offers certain affordances (potentials for action in relation to the actor, following Gibson, 1977), of which salient affordances are expected to be the most relevant (Norman, 1999). Affordances are the means for participation in social realms of activity as well as physical ones. Affordances are not deterministic: actors may appropriate affordances in particular ways to enable their participation. However, the availability and salience of affordances of designed artifacts influence these choices in ways that are of interest to us as researchers and designers.

\section{Data Requirements}

\section{What kinds of data are needed to address the research questions?}

In general, the kinds of data that are needed are process data concerning individual participants' manipulation of media and the availability of these manipulations to other participants, as well as 
some kind of "outcome" data that helps us select interactions that were fruitful from a learning perspective. These two requirements are discussed below, along with implications for data representations.

Ideally, the data would capture uptake relationships between media coordinations. Some data, such as reply relations in discourse media, make uptake explicit, but generally we cannot expect data to simply make the uptake apparent. People take up each other's contributions or traces thereof in ways that are often not explicitly recorded in the medium of interaction. Participants might reuse someone's phrasing, re-express their ideas, begin to attend to an issue because someone else raised that issue, react emotionally to a statement, etc. Uptake can be manifest in nonverbal forms, such as manipulating representational objects previously created in a shared workspace by editing, organizing or connecting them. Human action is contingent upon the "context" (physical environment, history of interaction, institutional and cultural-historical settings) in many subtle ways. Therefore the data should record sufficient information that enables us to identify various contingencies between media coordinations that may evidence the presence of "uptake."

The myriad of contingencies in human behavior can be overwhelming, and many will not turn out to be relevant to a given analysis. An analysis must be selective. Our strategy is goal directed: we identify particular outcomes of interest - such as participants coming to agreement on an explanation for a complex phenomenon, or a participant integrating several lines of discussion - and then work backwards to provide an interactional account leading to this outcome. The data requirement here is that there be some means of identifying outcomes worthy of this goal-directed analysis. In the context of educational research, we expect that the criterion will be some measure of or judgment concerning learning.

To date, we have not made much use of demographic, dispositional, or developmental data on participants. We have focused on what was available in the interaction being observed. (In theory, interaction projects back to birth and the prior interactions of others, but of course such data is not available: dispositional descriptions are convenient summaries of the future effect of prior interactions.) The lack of an explicit means for including such data may be a shortcoming of our approach to be addressed in future work, but for now it is a simplifying research strategy: to see what the interaction can tell us. Also, our approach does not exclude bringing in such considerations in interpreting the interaction.

The specific data required depends a great deal on the nature of the environment being studied. The nature of the data that comes from the environments we are studying is the first source of our analytic challenges, discussed next.

\section{Analytic Challenges}

\section{What are the biggest analytic challenges?}

The analytic challenges begin with the nature of the data itself: how do we make interaction apparent from the myriad of contingencies between media coordinations that are distributed across time, space and media? Then, because the data is multimodal we need to provide an account of nonverbal behavior. Our desire to scale up to larger data sets leads to questions of multi-scale phenomena. 


\subsection{The distributed nature of the data}

Some analytic challenges come from the setting. We have chosen to study technology affordances for intersubjective meaning-making in settings that (a) have multiple notational resources for interaction and (b) are spatially distributed and may be quasi-synchronous or asynchronous. Property (a) means that we need to construe activity that may be recorded in multiple log files or formats as unified activity, because interaction is distributed across all mutable media (Suthers, Hundhausen, \& Girardeau, 2003). We need a way to gather together data from various sources and derive an analytic artifact that enables us to "see" interaction more directly. Property (b) means that we cannot assume that the frame of reference is the same for everyone. We need to trace out activity with respect to individual frames of references and how they intertwine into phenomena at the group level.

\subsection{The contingent nature of human behavior}

Human action is contingent upon the "context" (physical environment and recent history of interaction) in many subtle ways. For example, even in the constrained environment of a threaded discussion it is not sufficient to consider the reply structure recorded in the media. A "posting" (message) can also be contingent upon other prior media coordinations in many ways. It can repeat lexical strings, re-use typographical conventions, or follow up on ideas of previous postings not limited to the one being "replied to." A posting can also be related temporally to the timing of other postings and (in an aspect of interaction often neglected in the study of threaded discussions) can also be temporally contingent upon the reading of other messages (Suthers, Dwyer, Medina et al., 2007a). The possible relationships get more complex in graphical workspaces, where for example the placement of a shape on the screen is contingent upon the prior placements of other shapes (Shipman \& McCall, 1994). How do we decide when the contingencies between media coordinations merit the appellation of "uptake" and therefore inclusion in the analysis of interaction?

\subsection{The meaning of nonverbal behavior}

Other analytic challenges are related to the meaning of nonverbal behavior. In addition to writing statements or labeling objects in natural language, users of a multimedia environment can manipulate and organize representations in ways explicitly and implicitly supported by the environment, such as linking objects or placing them in spatial arrangements relative to each other. When are such manipulations merely 'housekeeping' and when are they conceptually significant? Later in this paper we provide an example of how we uncovered conceptually significant manipulations of a graph representation of evidence.

\subsection{Selective attention to large data sets}

A fourth analytic challenge is related to our objective of scaling up interaction analysis from single sessions of dyads and small groups to larger groups, longer time spans and multiple media. We are able to record a large quantity of interaction data, but to what should we attend in our analysis to make our work tractable? Which interactions are meaningful? How do we ensure that this necessary selective attention does not leave out important observations or phenomena?

\subsection{Multi-scale phenomena}

Even in dyadic interaction, multiple scales of analysis are required. We can understand the moment to moment actions of each individual in the context of their environment, thread these 
actions into coherent accounts of individual trajectories of learning, and analyze interactions between trajectories that lead to meaning-making at the dyadic or small group level. As the time scale of such an analysis increases, we can observe the introduction or improvisation of new practices and their adoption and development by the group. As the number of persons involved in technology-mediated environments increase, phenomena that transcend the immediate interaction between individuals emerge, such as collective resources, practices and identity. The challenge is to not only identify phenomena at a given temporal or social scale, but to find relationships between phenomena across scales.

\section{Analyzing Distributed Interaction with Contingency Graphs}

Which challenges have you successfully addressed?

Our approach to analysis addresses some of the above challenges through an abstract transcript representation that we call "contingency graphs." This section describes contingency graphs, summarizes how we use them to address the analytic challenges, and gives an extended example.

\subsection{Contingency Graphs}

We have developed an abstract transcript format, the "contingency graph," that captures interaction in a medium-independent manner that yet admits of annotations concerning media properties. This notation enables us to gather together activity that is distributed across media (and hence data sources) and across participants into one analytic artifact. A contingency graph consists of vertices (nodes) that represent media coordinations and directed arcs (links) that represent contingencies between these coordinations. Multiple log files from multiple sources of data are merged into the contingency graph, retaining pointers back to the original data but enabling us to have a single abstract transcript that gathers together all potential interaction into one artifact that can be visualized and/or searched through computational processes.

\subsubsection{Vertices: Media Coordinations}

The media coordinations represented by vertices may include any manipulation of the medium that is available to us, including (for example) not only the creation of media inscriptions (e.g., posting a message, making an object in a workspace), but also manipulation of those inscriptions (e.g., moving objects closer to each other) and perception of those inscriptions (e.g., opening a message to read it). The graph also records computer-initiated events such as the display of inscriptions that come from other participants in an asynchronous environment, or events initiated by the technological infrastructure itself.

\subsubsection{Arcs: Contingencies}

A contingency relationship holds when one or more events enable a subsequent event. The term "contingency" is chosen to indicate a sense of enablement in which human action draws upon but is not necessarily determined by elements of the environment, as discussed in section 4.2 and further below. Contingencies are represented in an acyclic directed hypergraph as hyperarcs (directed hyperedges) between events. Each arc points backward in time from a single origin to one or more destinations. For example, in Figure 1, event E3 is contingent on event E1, and E4 is contingent on events E1 and E2.

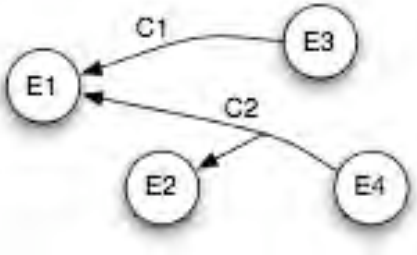

Figure 1. Contingency relationships 
Over the past two years, we have done considerable work exploring types of contingency relationships between events (Suthers, 2006a; Suthers, Dwyer, Medina et al., 2007a; Suthers, Dwyer, Medina, \& Vatrapu, 2007b; Suthers, Dwyer, Vatrapu, \& Medina, 2007), including media dependencies, temporal and spatial proximity, representational similarity, and semantic overlap.

Contingencies are most easily identified through similarities in events. The most straightforward approach is to construct contingency arcs between events that involve the same media entity or dependencies between media entities. For example, the events of opening and replying to a message are dependent on the event of creating a message, and the event of linking to or annotating a media entity depends on its prior existence.

In synchronous interaction, temporal proximity also implies relevance, such as in the typical reply structure of conversation (Sacks, Schegloff, \& Jefferson, 1974). People also exploit spatial proximity and representational similarity to manage interaction and express association (Dwyer \& Suthers, 2006; Shipman III \& McCall, 1994). For example, if a representational element is given the same appearance as other elements (e.g., same color, location, or label), we construe the change in appearance as contingent on previous uses of those visual attributes (e.g., adding an element to a group is contingent on the group's prior existence).

Tracing semantic overlap is more difficult. We can partially trace ideas by tracing the artifacts that express them, but actors may "transcribe" ideas to other artifacts, such as through quoting practices (Barcellini, Détienne, Burkhardt, \& Sack, 2005). More problematically for the analyst, ideas can be taken up and re-expressed in different ways. It is precisely these kinds of semantic transformations that are of greatest interest when studying (for example) the production of new knowledge in technology-mediated social networks.

\subsubsection{Addressing the Challenges}

Contingency graphs address the distributed nature of the data by providing a single analytic artifact into which the data is gathered. Clearly, they help begin address the contingent nature of human behavior by providing an explicit representation of contingencies so that there is a basis for deciding which contingencies are relevant, using both automated tools and human judgment. (Many approaches to coding discourse relations allow the analyst to simply assert the relation without requiring that the analyst specify the contingencies on which this judgment was based, so do not support conversations about the choices made by analysts.)

The challenge of understanding the meaning of nonverbal behavior has two aspects: selectional and hermeneutic. Contingency graphs can only help us with the selectional problem of identifying nonverbal behaviors that merit closer examination to determine whether they are interactionally meaningful. As will be illustrated in the next subsection, some nonverbal behaviors of interest result in patterns in the contingency graph. Unlike separate log files, contingency graphs that unify all behaviors provide a uniform way to see patterns of nonverbal manipulations and situate them in the context of verbal behavior. This turns out to be important because some patterns in nonverbal behavior are found in relation to verbal behavior. To address the second, hermeneutic problem one must go to the data in the original media formats and make interpretations. The contingency graph helps focus this effort.

We address the problem of selective attention to large data sets by using goal-directed search in the contingency graph (e.g., tracing back from an interesting learning outcome), by using automated tools for finding relevant events and pathways in the graph, and through visualizations 
of the graph structure. Finally, we believe that contingency graphs will help address multi-scale phenomena through computational tools that enable analysts to find patterns at larger scales and relate them to aggregate phenomena. Our following example illustrates multi-scale analysis at the scales of episodes in a session down to micro-analysis. Extensions in the other direction, to be explored with our online community data, are still pending.

\subsection{Analyzing distributed interaction with contingency graphs}

This section describes a recent analysis using the contingency graph. It illustrates how we have begun to confront the challenges of working with records of distributed data and activity, finding significance in nonverbal actions, selectively attending to particular aspects of the data, and moving flexibly across multi-scale phenomena. The session we analyzed will be described next, followed by a description of the log file and video data. This section ends with detailed account of the analysis revealing a qualitative explanation of convergence based on verbal and nonverbal interaction.

\subsubsection{Source of Data}

The case study presented here illustrates a pattern of interaction between two individuals engaged in a joint-problem solving exercise while using a shared networked workspace environment (Figure 2). The individuals were participating in an experiment described in (Suthers, Vatrapu, Medina, Joseph, \& Dwyer, in press). Using informational materials we provided in the workspace, the two participants (P1 and P2) worked to identify possible causes of a disease in Guam, ALS-PD (Amyotrophic Lateral Sclerosis-Parkinsonism Dementia complex). The session took place

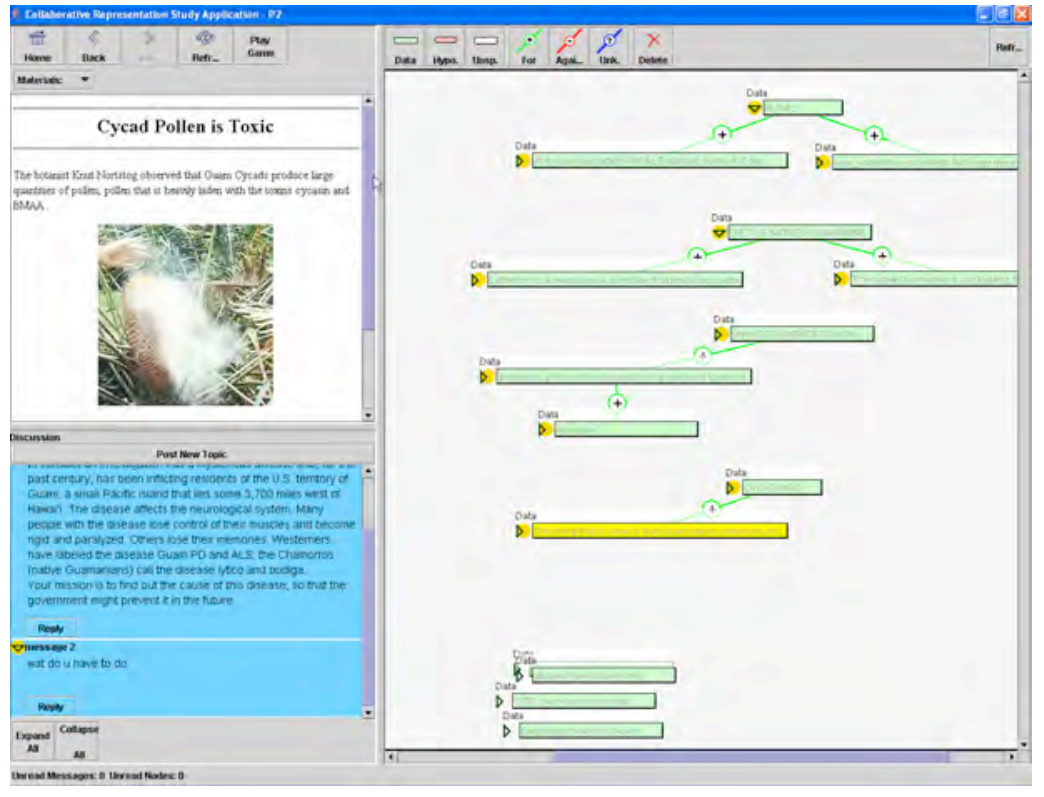

Figure 2. Information source (top left), threaded discussion (bottom left) and evidence map (right) over the course of approximately two hours. Participants were at different locations, and interacted in an environment that included a graphical evidence map and threaded discussion. Each participant's view of the shared environment was updated using a software protocol that enforced asynchronous interaction by distributing respective workspace changes at intermittent times during the interaction (participants were also able to manually request updates by selecting a refresh button). We undertook the analysis to account for ways in which participants both converged and diverged in their interpretations of causes of ALS-PD, by tracing out sequential patterns of representational practices enacted within the workspace. The analysis highlights an evolving transformation of a collaborative representational practice. These practices and the artifacts left in their wake provide an explanation for the conceptual convergence and divergence in the conclusions expressed by each participant. 


\subsubsection{Log File Description}

The software used by these participants provided both threaded discussion and graphical evidence mapping tools. All actions performed by participants on and through the software media were logged. In addition to actions, network activity was logged to record when events recorded on each participant's machine were received and rendered on the other. With regard to the use of log files in this analysis, all acts have a corresponding media coordination event in the $\log$ file, can be attributed to a participant, can be identified in terms of the media object or objects implicated in the act, and are time-stamped relative to the machine on which the act was performed. Also, all network exchanges between participants' machines have a corresponding event in the log file. In addition to log files, we have a video record of each participants' screen, along with synchronized web-camera video of participants' faces, using Morae ${ }^{\mathrm{TM}}$ software.

\subsubsection{Contingency Graph Construction}

In this analysis, the basic contingency graph of log data was generated by iteratively relating pairs of log events based on the following criteria (see Figure 3 for an example):

- If two events share an object id (an artifact is edited, moved, etc.) then the later event is contingent upon the prior event.

- The event of linking two objects in the evidence map is contingent upon the most recent prior events available to the participant that modified the objects.

- The event of posting a discussion message is contingent on the event that created the message.

\begin{tabular}{|c|c|c|c|c|c|}
\hline \multirow[b]{2}{*}{ HOST LOG ID } & \multicolumn{4}{|c|}{$\begin{array}{l}\text { M1P1's Machine } \\
\text { Log }\end{array}$} & \multirow[b]{2}{*}{$\ldots$} \\
\hline & $\begin{array}{l}\text { REMOTE LOG } \\
\text { ID }\end{array}$ & HOST TIME & HOST ID & CREATOR & \\
\hline 16589 & 17526 & $00: 39: 06$ & MIPI & M1PI & $\ldots$ \\
\hline 16593 & 17446 & $00: 41: 07$ & M1PI & M1P2 & $\ldots$ \\
\hline 16606 & 17459 & $00: 41: 07$ & M1PI & M1P2 & $\ldots$ \\
\hline 16618 & 17474 & $00: 41: 07$ & MIPI & M1P2 & $\ldots$ \\
\hline 16658 & 17514 & $00: 41: 07$ & $\mathrm{M} 1 \mathrm{PI}$ & M1P2 & $\cdots$ \\
\hline 16678 & 17625 & $00: 47: 04$ & MIPI & M1P1 & $\cdots$ \\
\hline
\end{tabular}

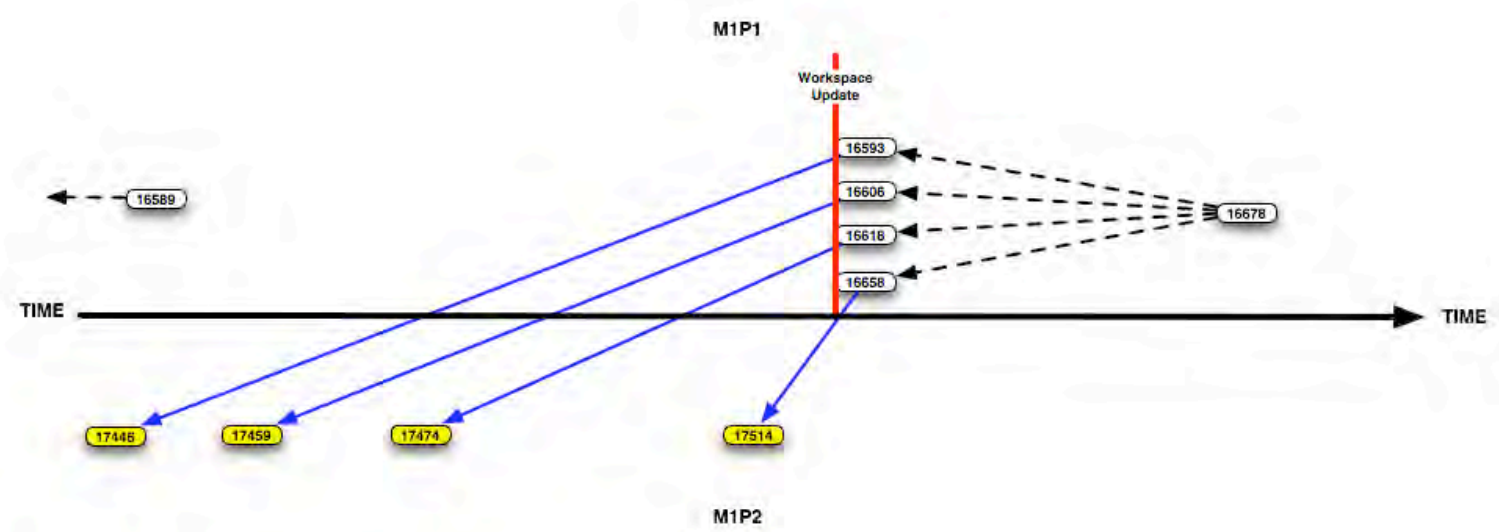

Figure 3: Example log file segment (after pre-processing) and corresponding contingency graph representation. Media coordinations above the timeline were by Participant 1, and below the timeline were by Participant 2. 
- If a discussion message contains a hyperlink to a graph object, then the message event is contingent upon the most recent event available to the participant that modified that graph object.

Many other contingencies are available, but the above provided a sufficient starting point for the analysis. A visualization tool (Omnigraffle ${ }^{\mathrm{TM}}$ ) was used to render the contingency graph. See Figure 4 for an example.

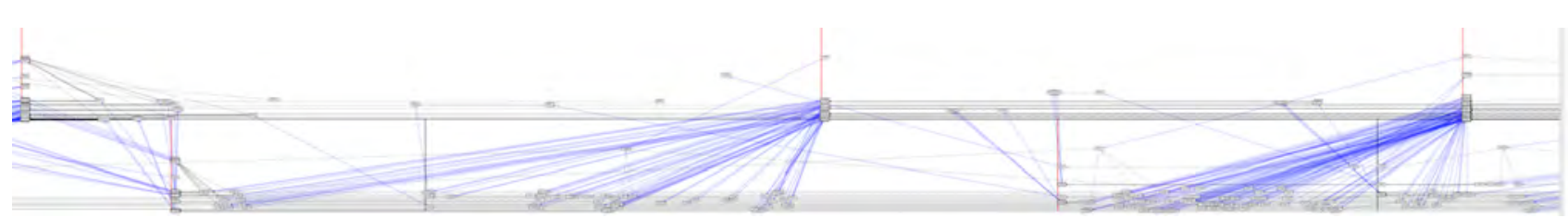

Figure 4. A 20 minute segment of an automatically generated contingency graph.

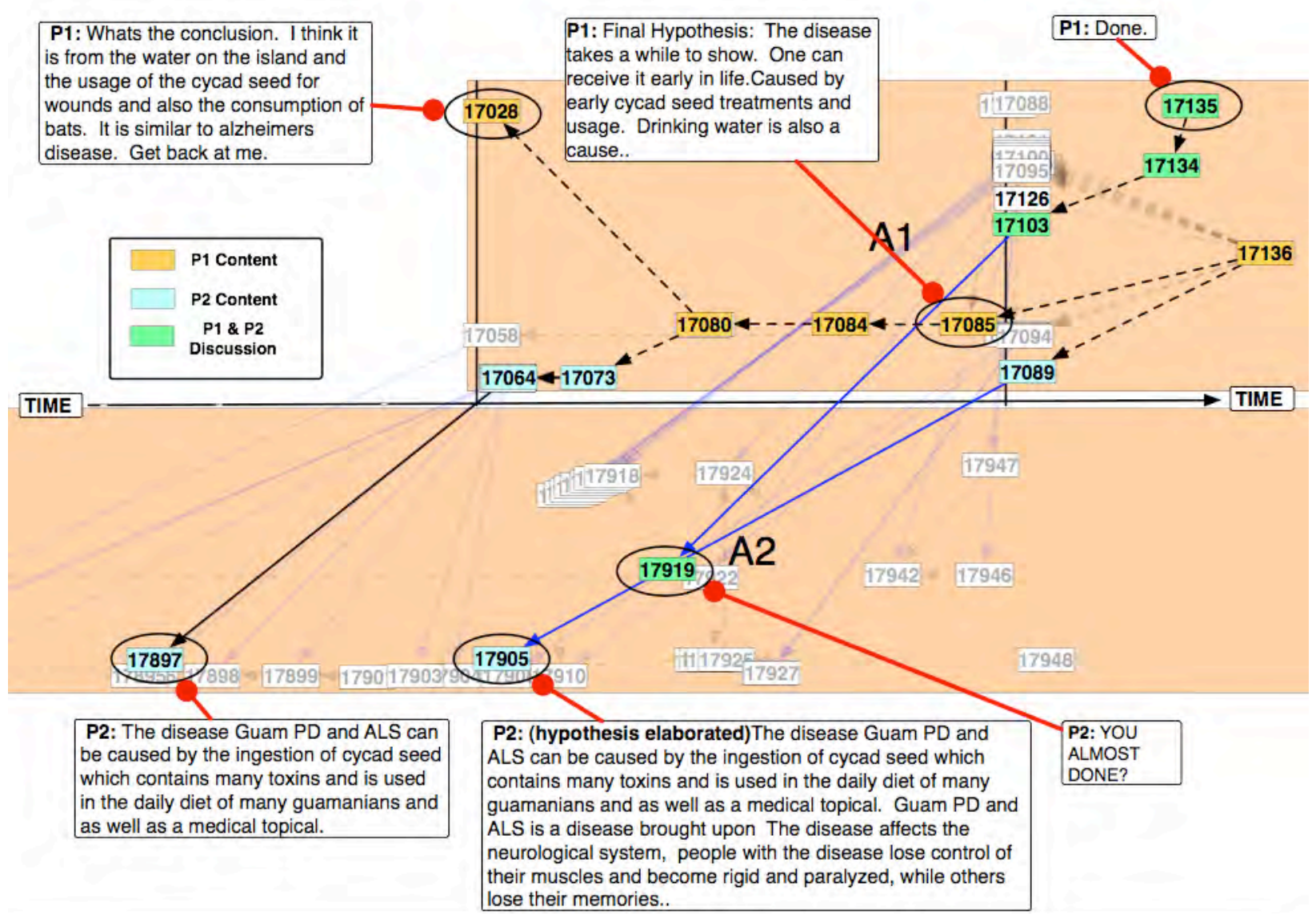

Figure 5. Subset of contingency graph showing the conclusions expressed by both participants

\subsubsection{Contingency Graph Analysis}

\subsubsection{Selective Attention: Identifying Convergent Conclusions}

The analysis begins with an important reference point in the interaction, a sequence of activity in which both participants express conclusions concerning the possible causes of ALS-PD. This episode takes place in a time span of approximately 10 minutes towards the end of the session. 
This portion of the record was selected as a starting point because it presented an opportunity to understand the conclusions expressed by participants as they began to summarize their ideas. The beginning of this episode is indicated by P1's prompting for a conclusion ([17028] in the top left of the contingency graph of Figure 5). P1 makes this request using a discussion posting. Despite the fact that $\mathrm{P} 2$ does not read the message (P2 did not initiate further requests for workspace updates), it indicates P1's plans to initiate a negotiated conclusion. This is evidenced by a subsequent act that proposes a "final" conclusion [17085] incorporating formative elements of P2's concluding work, [17897], which coincidentally begins and is concurrently developed by P2 [17905], at approximately the same time as P1's (unread) request. The episode ending is negotiated when P2 asks whether P1 is done [17919]. P1 reads and responds by stating; "Done" [17135] then immediately makes a final "For" link between P1's and P2's hypothesis node [17136]. The content analysis of this segment of the interaction reveals an instance of convergence on "cycad usage" and an instance of divergence on "aluminum" as a causal agent for the disease. Based on this analysis, two traces were initiated in the complete contingency graph to highlight those nodes that contained or were related to references to aluminum and cycad.

\subsubsection{Tracing: Subgraph Building Reveals Non Verbal Interaction Pattern}

One of the consistent concepts indicated in P1's argument during Segment A1 (Figure 5) is that "drinking water" is one possible cause for the disease. An attempt to build an account of this concept through the interaction history began by forming a query (input into a prototype tool) in order to highlight acts that reference that text string and the contingencies between those acts. The graph revealed references to "drinking water" that were included in the information provided to P1 in relation to aluminum as a potential cause of the disease. A second query was invoked to capture acts that also referenced aluminum, extending the trace. The resulting contingency graph is summarized schematically in Figure 6.

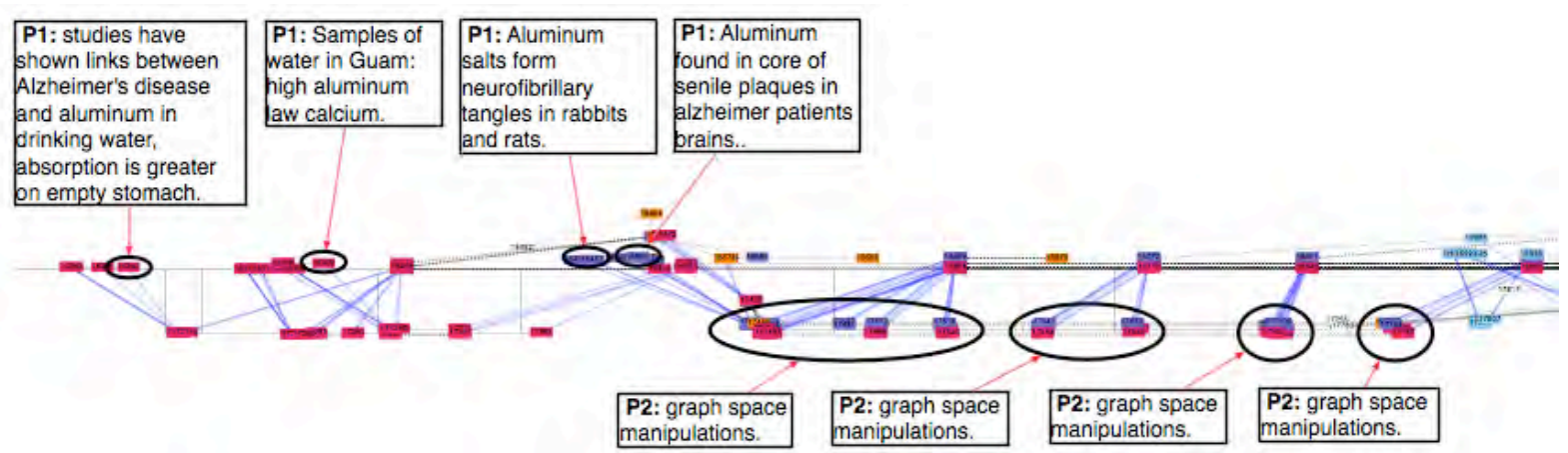

Figure 6. Information sharing by P1 followed by systematic graph manipulations by P2 during the first hour of the session.

The graph revealed that in two particular instances P1 shares information with P2 related to the contamination of drinking water by aluminum. P2 performs a series of moves evidenced by clumps of move events in the graph. These acts by P2 do not contain linguistic responses; only a series of moves (drag and drop acts) in the graph space. This pattern is consistent throughout the remaining portions of the session. The trace shown in Figure 6 could indicate that P2 is moving nodes around in order to see them, or to get them out of the way: dragging and dropping of graphical objects for these reasons is frequent. In this case however, the periodic-like pattern and 
density of P2's series of movements suggested more deliberate activity and induced us to explore the video record for these episodes to determine how these non-verbal manipulations might have influenced the interaction, especially with regard to the convergence identified above. The video shows that P2 is not randomly moving nodes around, but performing a series of graph space reconfigurations to organize information previously shared during the session. After P1 contributes new information, P2 moves nodes to create spatially distinct groups that provide conceptual delineation. In addition to this spatial organization, both participants create links between nodes within groups that further clarify their inclusion in the group. (Their work will be illustrated in detail in the next section.)

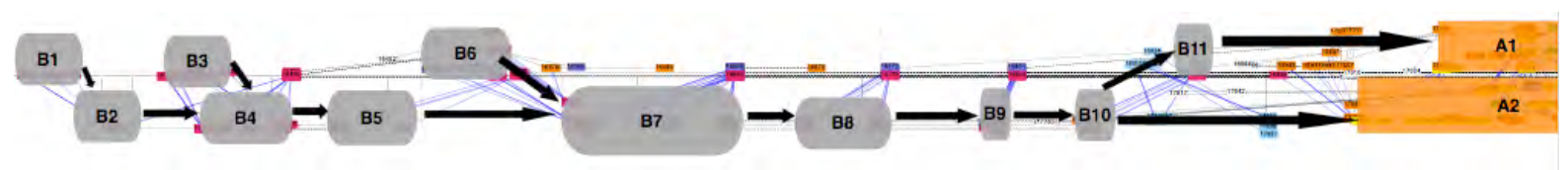

Figure 7. High level view of uptake over the entire session.

Figure 7 illustrates the trace listed in Figure 6 at a higher level of abstraction, as a series of uptake relations between episodic segments. Beginning at the left, P1 shares information containing a reference to aluminum in water as a contaminant in the first two segments [B1 \& B3]. The third information-sharing event by $\mathrm{P} 1$ contains two references that correlate aluminum and neurological symptoms of ALS-PD [B6]. The reaction to the three sharing acts by P2 is shown as episodes of graph space manipulations [B2, B4, B5 \& B7-10]. Intersubjective uptake is indicated by P2's visual transformation of the shared information nodes and is followed by a series of intrasubjective transformative acts on the part of $\mathrm{P} 2$, who continually appropriates the relation-indicating power of the graphical nodes. The fact that there is very little related action on the part of $\mathrm{P} 1$ during these acts indicates that $\mathrm{P} 2$ is accountable for subsequent transformations. As shown on the far right of the diagram, intersubjective acts again occur as the concluding work segment discussed above, is initiated [A1 \& A2]. Closer examination of selected video segments using the contingency graph as an index, reveals a purposed appropriation of the graph space.

\subsubsection{Micro Analysis: Indexing Video to Correlate Individual and Social Phenomena}

The patterns represented in the contingency graph provided frames of reference and direct pointers, via timestamps, to relevant locations in the video record. More significantly, this framing made the interrelation between the two separate video streams salient for determining the emergence of a shared representational practice.

As discussed above, there is a visible distinction in the participants' respective roles with regard to media coordinations: P2 does more graph related work and P1 does less action within the graph but expresses verbal articulations of hypotheses throughout the interaction. Their implicit role negotiation during joint problem solving developed early in the session and carried across different conceptual trajectories (Medina \& Suthers, 2008). For example, participants diverge on "aluminum", but converge on "cycad usage" as a cause of the disease. Next we describe key aspects of cycad convergence and how it is interactionally managed through representation. 


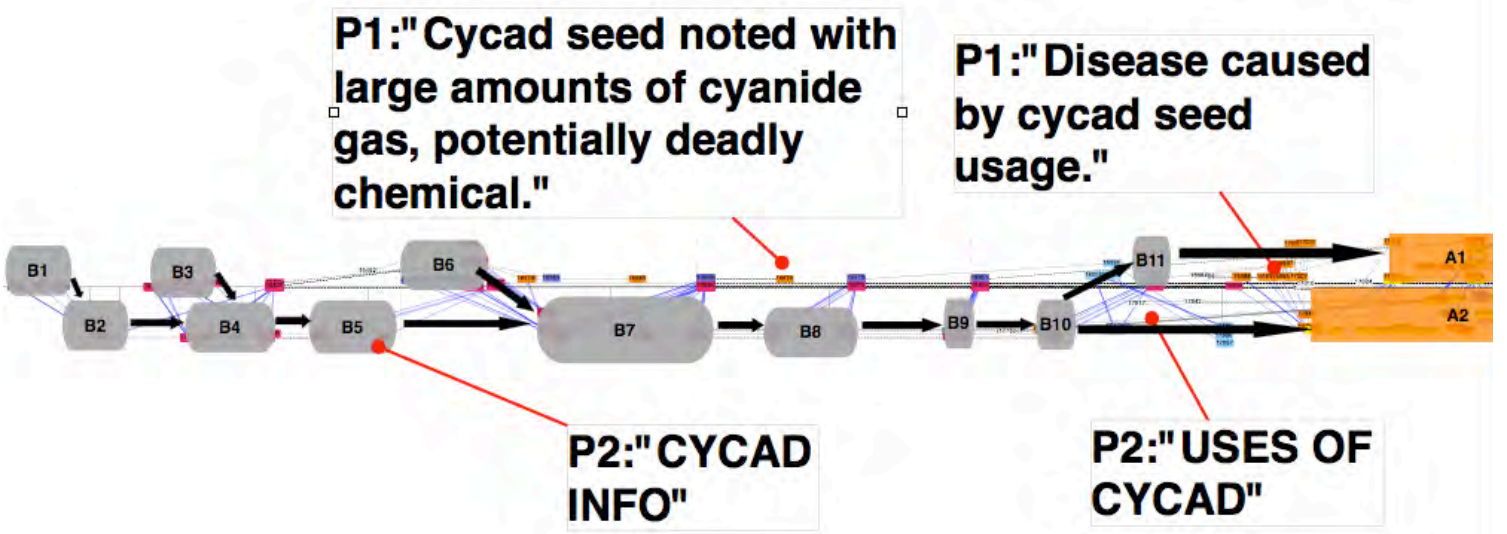

Figure 10. Screen video is indexed in contingency graph (statements reference figures 9,10 and 11)

Figure 10 shows the sequential introduction of cycad information and Figure 8-11 show how this was done in the graph space. Following his own representational convention, P2 positions the label, /CYCAD INFO/, and three related data nodes into a configuration similar to other conceptually organized subgraphs (Figure 9). Figure 10 shows a subsequent act on P1's machine where she introduces a data node containing information about cycad seeds. (Time has elapsed, so P1's screen reflects the ongoing work of the two participants.) In this context, a cycad related node is created and positioned in a somewhat arbitrary location with regard to the ongoing visual grouping. On receiving an update from P1 containing the cycad data, P2 reads the contents of the node, drags the node to a "member" position of the cycad conceptual grouping, and creates a "+" link between the node and the /CYCAD INFO/ hub, further expressing its group membership (Figure 11).

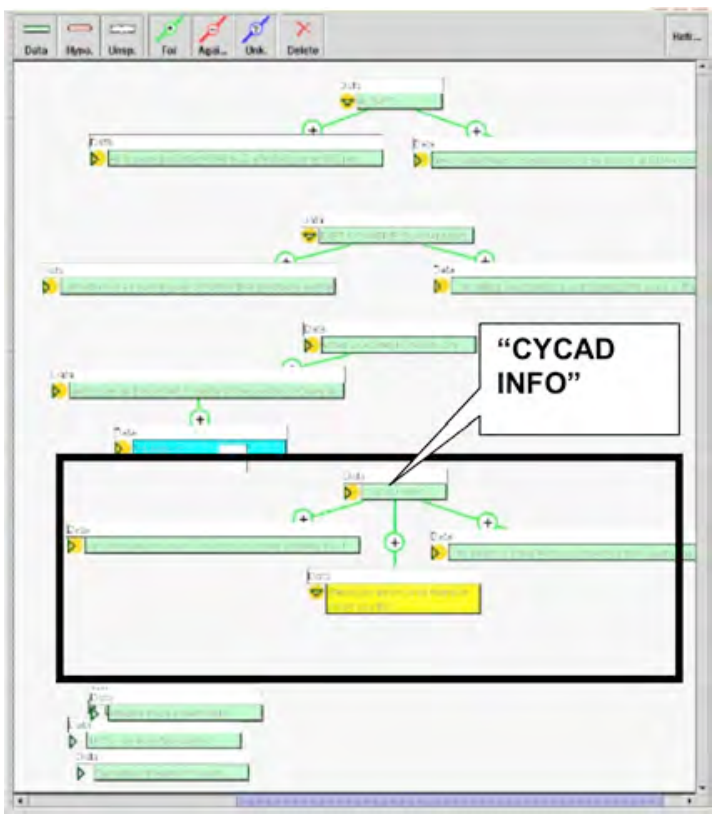

Figure 8. P2 creates cycad representation

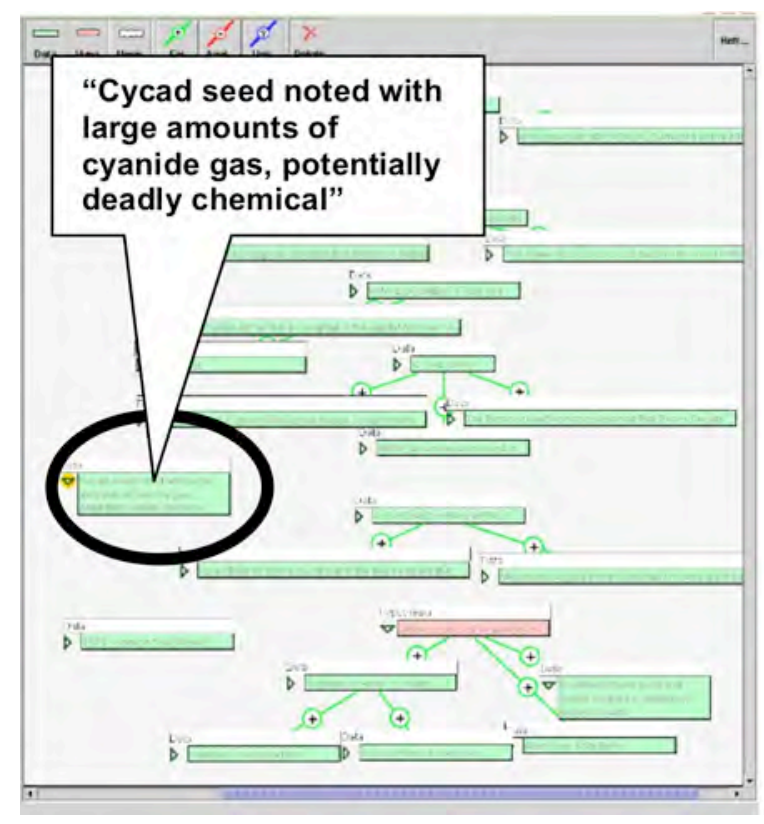

Figure 9. P1 creates a cycad data node 
Subsequently, each participant brings "cycad usage" forward in distinct ways. P1 articulates cycad salience through a statement placed in a Hypothesis node, /Disease caused by cycad seed usage/ (Figure 12, left side), while P2 posts a short "themed" node expressing /USES OF CYCAD/ (Figure 12, right side). Each participant without knowledge of the other performs these respective acts. They coincidentally indicate cycad usage at approximately the same time. In addition to posting her hypothesis node, P1 integrates it into the /CYCAD INFO/group configuration by creating four links to supporting data. P2 also groups and links data nodes to their expression ("USES OF CYCAD"). It is a mutual appropriation of a grouping practice. $\mathrm{P} 1$ and $\mathrm{P} 2$ both begin wrapping up their work within five minutes after this episode and thus initiate the concluding work episode presented

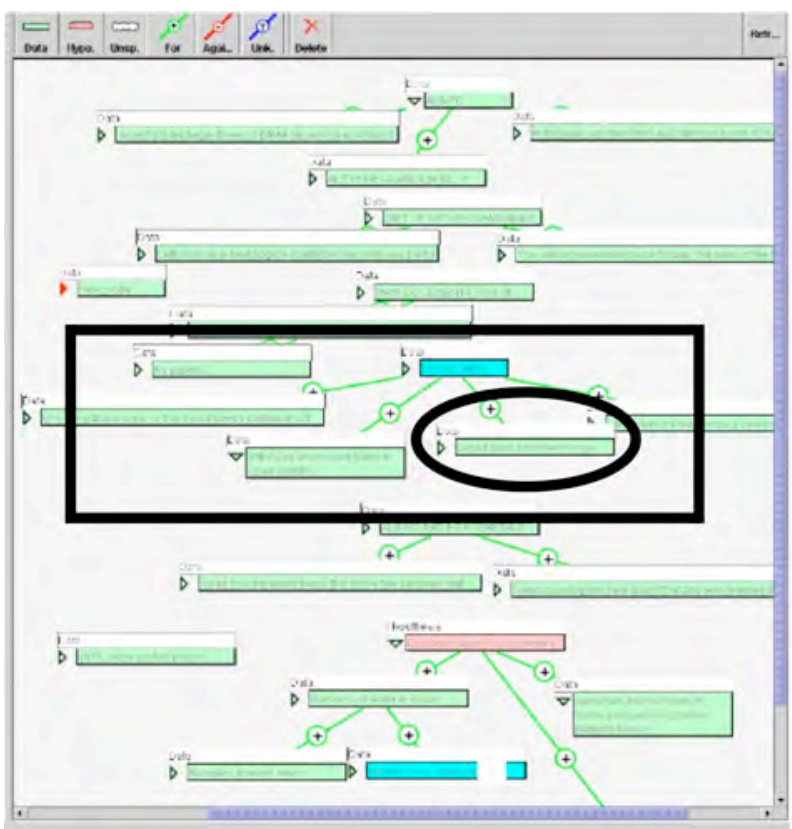

Figure 11. $P 2$ receives cycad data node from $P 1$ (Figure 10) and repositions and links into cycad group (Figure 9) above.
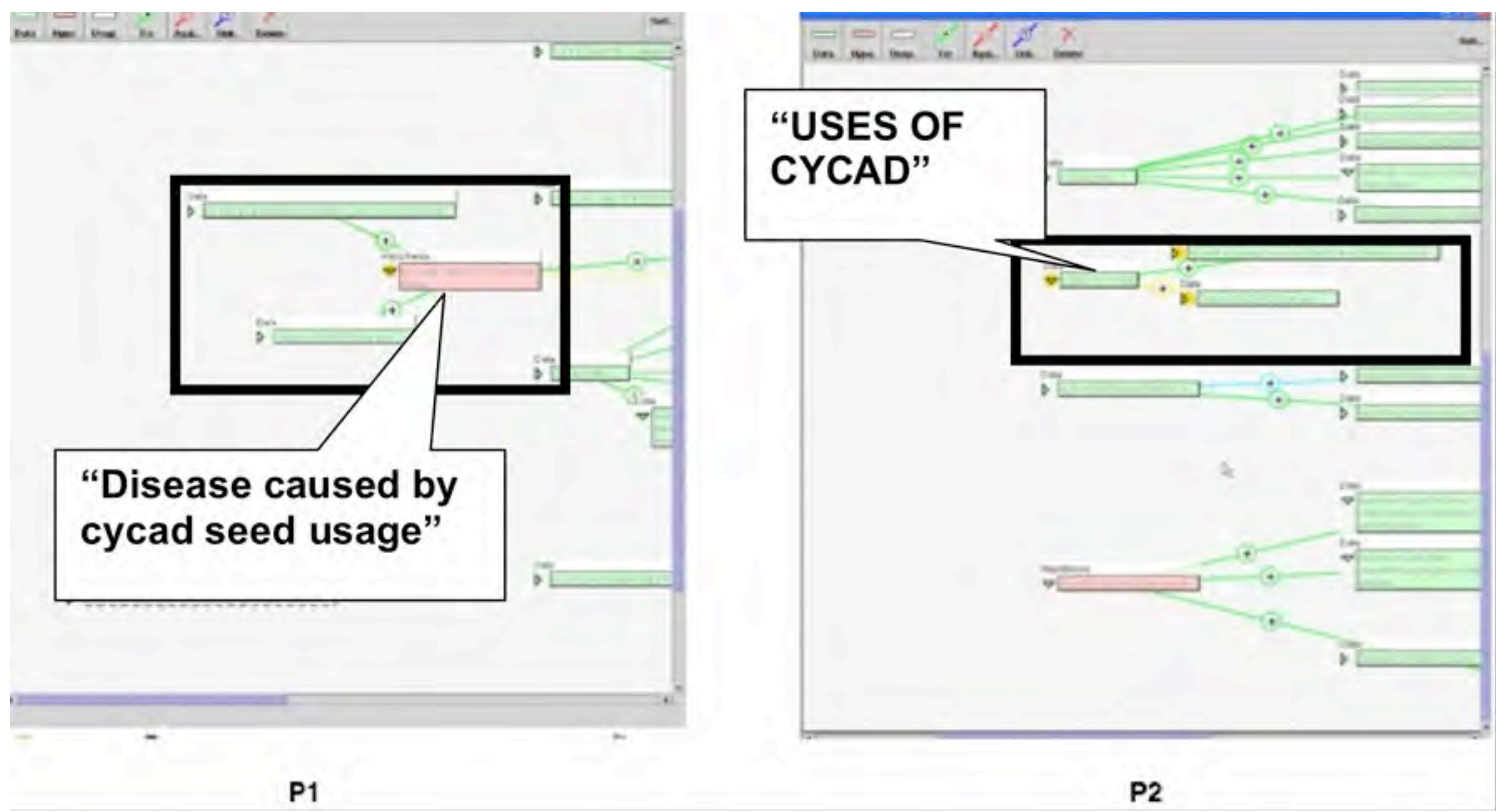

Figure 12. $\mathrm{P} 1$ and $\mathrm{P} 2$ articulate new cycad groupings independently. (P2 has reorganized the graph into a horizontal layout format.)

\subsubsection{Analytic Rationale for using the Contingency Graph}

The use of the contingency graph during this analysis supported flexible transitions between identification of macro interaction patterns and micro analysis of a series of graphical 
manipulations. As illustrated above, starting with analysis of short segments of interaction, it was possible to identify molecules of ideas. Using the contingency graph as an artifact encompassing the entire recorded interaction, it was then possible to fix, in time, individual and joint conceptual development. This, in turn, provided additional points that induced deeper investigation, transitioning back to analysis of micro segments. Understanding interaction requires macro and micro understandings by necessity. The contingency graph floats between the two.

\subsubsection{Scaling Up}

Beyond the analytic applications we have already attempted, we envision using the contingency graph to scale up interaction analysis to larger data sets. For example, in order to understand learning in socio-technical networks (e.g., "online communities"), we would like to explain how ideas develop as they move though social networks; how people acquire their roles in social networks; and how technological artifacts mediate these transformations of people and ideas as the artifacts themselves move and are transformed in social networks. We are currently doing preliminary work in tracing out the pathways by which new social relationships are formed (Joseph et al., 2007). It is our intention to generalize the contingency graph to an abstract transcript representation that supports a variety of analyses in larger scale social networks.

\section{Remaining Challenges}

What challenges still need to be addressed?

In the previous section, we provided our vision for how the approach might be generalized and scaled up. Work remains for each of the challenges previously listed.

\subsection{The distributed nature of the data}

The contingency graph offers a place to gather distributed data together into a single analytic artifact. In order to realize this benefit, we need to define the contingency graph as an abstract data type with an application program interface (API), and use that API to write translators that import various log and sensor formats into the data type.

As a single representation of interaction, the contingency graph potentially enables analysts to "see" distributed interaction. However, the graph itself is an abstract structure. In order to realize this potential tools are needed to visualize and query that structure in useful ways (see Selective Attention, below).

\subsection{The contingent nature of human behavior}

The contingency graph obviously helps address this challenge by making contingencies explicit. In another sense the contingency graph makes the problem worse by forcing us to deal with these contingencies, but the fact that we are working with complex and sometimes circumstantial evidence cannot be sidestepped. Contingencies in the data model must include documentation of the evidence used to generate them, and analysts must be able to repudiate automatically identified contingencies as well as manually specify new ones.

We need to recognize that the record of proximal interaction may not capture all of the relevant contingencies, as some are based on events prior to the recorded interaction. An extension to our work could provide a means of introducing noninteractional data such as dispositions and prior knowledge into this analysis. Epistemological as well as methodological 
issues will need to be addressed, as this move involves combining two traditions. In analogy to Latour's (2005) distinction between the "sociology of the social" and the "sociology of associations," dispositions and other prior individual differences belong to the "psychology of the psychological," while our approach is a "psychology of interactions" (see also Edwards, 1997).

\subsection{The meaning of nonverbal behavior}

The primary technical challenge in analyzing nonverbal behavior is to make the relevant media accessible. Ideally we would have an integrated environment in which one could access and view the media manipulations (graphical workspace manipulation, message postings, wiki edits, etc.) on demand. The contingency graph can serve as a sort of index and player synchronization device in such an environment, as well as being an analytic artifact in its own right.

Those technical accomplishments would make the relevant behavior available, but the hermeneutic problem would remain. By making the behavior available in a form that is amenable to inspection, collaborative interpretation including participants as well as other researchers becomes easier.

\subsection{Selective attention to large data sets}

This open technical problem includes the development of search, query and visualization tools aided by information filters. Strategic solutions are specific to the purposes of the analysis.

\subsection{Multi-scale phenomena}

Some of the most interesting challenges lie here. There are theoretical as well as empirical challenges.

The nature of our data has led us to consider two questions of scale. One is the extent to which sequential analysis of interaction of the sort that is normally associated with microanalysis of face-to-face data (e.g., conversational transcripts or video) can be extended or scaled up along several dimensions: to interactions that are distributed across space (including locations and media), and across time (including asynchronous interaction); to include larger numbers of participants; and to longer time spans. We view this problem partly as an information processing and human-computer interaction problem: gathering the appropriate data and making it accessible to analysts in representations that make previously occult processes plainly visible. But we also see a theoretical problem concerning the adequacy of description: to what extent does tracing out lines of interaction remain to be a productive level of description, and at what point is it useful to shift to an aggregate level of description?

The other question concerns how implications for design can be drawn as we abstract away to aggregate phenomena. Even the largest aggregate phenomena fundamentally derive from individuals interacting with technological environments moment-to-moment, and making decisions at each moment that contribute to the aggregate results. These decisions are the point of contact between design of the technological environment and construction of the social reality. We are working on a theoretical account that bridges from these moments of experience and decision to other such moments at other times and places, aggregating to phenomena termed "social". Beginnings are in (Joseph et al., 2007). 


\section{A Generalized Framework for Analysis}

More recent work seeks to generalize our framework for conceptualizing interaction. Interaction is an ongoing process in which multiple actors exchange information and construct new knowledge using multiple semiotic tools. These processes encompass the individual-cognitive and social-technical realms, and include artifact-mediated coordinations within and between these realms (Hutchins, 1995; Kaptelinin \& Nardi, 2006; Latour, 2005a). Our generalized model abstracts elements of these processes, identifies sequential relationships between these elements, and examines how these sequences are reflected in and constrained by the evolving social network structure. Our methodological hypothesis is that much useful analysis can be done by tracing out the movements, confluences and transformations of actors, ideas, and artifacts. We unpack this statement below.

\subsection{Trace actors, ideas, and artifacts}

Actors, ideas, and artifacts are the essential elements of any activity — actors express and interpret ideas via the artifacts they produce. We interpret each of these broadly.

\subsubsection{Actors}

We need to trace actors as they move through socio-technical environments because we are interested in how people utilize the resources of these environments, form relationships with other people, form larger networks of varying degrees of cohesion, and collectively create new value for their communities. Actors are primarily individuals, but we will sometimes wish to analyze in terms of collections of persons such as social networks discovered in the data (Cai, Shao, He, Yan, \& Han, 2005; Latour, 2005a) or groups defined by an identity marker (e.g., gender, age groups, institutional affiliations) (Cohen, 1985; Donath, 1998; Tajfel \& Turner, 1986). Software components may also be analyzed as actors in a limited sense (Latour, 2005a).

\subsubsection{Ideas}

We need to trace ideas as they move between people via artifacts because we want to know how ideas propagate in a community and how ideas are constructed in interaction. What counts as an "idea" can vary widely depending on the purpose of analysis. Ideas must be communicated through some medium. In online environments, actors make ideas accessible to one another via electronic artifacts that can themselves be traced.

\subsubsection{Artifacts}

We need to trace artifacts because we want to know how the socio-technical environments we design influence the other processes. For example, we may want to know how artifacts persist expressions of ideas in a manner that they will be found by those who care to take them up. Artifacts are any digital objects created as a result of human activity. Obvious examples include chat or discussion messages, documents, web pages, and URLs that reference them. Sometimes we need to analyze in terms of an aggregate artifact, such as a discussion forum or a workspace that includes multiple artifacts of a finer granularity. Other artifacts are created indirectly by activity. For example, the action of accessing a web page or reading a message may increment a counter that is displayed as a "hit counter" or read activity. 


\subsection{Trace movements, confluences, and transformations}

Process analyses are concerned with the movements, confluences, and transformations of these entities over the course of the interaction.

\subsubsection{Movements}

An analysis may hold one type of entity (actor, idea, artifact) constant and follow its movement with respect to changes in the other two. We can trace the following:

- How one person contributes or experiences multiple ideas and multiple artifacts

- How one idea can be represented in multiple artifacts and experienced/expressed by multiple people

- How one artifact can be perceived and modified by multiple people and contain multiple ideas

\subsubsection{Confluences}

Analyses can also trace how entities aggregate or intersect. To identify social networks, we would trace persons to find aggregations where their trajectories intersect via a shared set of artifacts (such as a discussion forum). For example, an analysis directed at finding boundary objects (Star, 1990) may begin with persons in a given social network, identify artifacts they have created, and then follow these artifacts to see whether they are taken up by persons in another social network. Such a trace follows a grammar that crosses two intersections: actor/artifact and artifact/actor. Searching for this grammatical pattern can be automated.

\subsubsection{Transformations}

Analyses that seek to identify and explain how ideas are constructed in a community are good examples of analysis of transformations. One would trace the origination and transformation of ideas as they are expressed by multiple people in persistent artifacts that are then taken up and transformed by other people, as we have done in our own work (Suthers, 2006a; Suthers, Dwyer, Medina et al., 2007a). The transformation of artifacts may parallel the transformation of ideas, but the two are not identical, as people take up ideas and express them in new artifacts. One might also trace transformations of persons through analysis of their postings and augmented by other sources of data.

In reality, we are almost always tracing movements, confluences and transformations simultaneously, because the confluences of trajectories of movement are the places to look for transformations.

\section{Conclusions}

How does addressing these analytic challenges advance our understanding of how people learn in technology-mediated environments?

Voluminous literatures exist for research that explores the relationship between pre-conditions or manipulated variables and learning outcomes, while black-boxing the processes by which this learning was accomplished. Such research is strong for hypothesis testing but weaker for discovery. As technological innovations proliferate and people's practices adapt to and adopt these innovations, we need to discover what is happening rather than to try to confirm what we have already guessed. This can be done only by making interaction visible: a challenge in complex and distributed environments. We have outlined an approach that "gathers together" interaction and makes possible tools that make it visible. We hope that the foregoing discussion 
has made clear how this approach enables a given analyst to understand learning in technologymediated environments.

But there is a larger advantage to be realized as well. Progress in any scientific discipline requires that practitioners share conceptual vocabularies. Major advances in other scientific disciplines have been accompanied with representational advances, and shared instruments and representations mediate the daily work of scientific discourse. Similarly, researchers studying learning in distributed and networked environments need shared ways of conceptualizing and representing what takes place in these environments to serve as the common foundation for our scientific and design discourse. Presently our community has neither a common representation of data nor a shared vocabulary to discuss it, so it is difficult to build on each other's work or to take advantage of the analysis tools built by different researchers. An abstract transcript format that captures relevant aspects of interaction in diverse media can serve as the basis for shared vocabulary when communicating with each other and for development and sharing of the software tools that are critically needed to scale up our analytic work. Also, we intend that the abstract transcript be sufficient to support the full range of analytic methods (ethnographic, sequential, statistical, etc.) consistent with the basic conceptual framework outlined in this paper. To the extent that we succeed, the contingency graph can also serve as a boundary object for discourse between disciplines that are addressing similar problems. A common abstract transcript can support and bridge between multiple theoretical perspectives as well as facilitating the application of different analytical methodologies and tools to complex data sets.

\section{Acknowledgements}

The approach to analysis described in this paper was developed over several years in collaboration with Nathan Dwyer and Ravi Vatrapu. This work was supported by the National Science Foundation under CAREER award 0093505.

\section{References}

Barcellini, F., Détienne, F., Burkhardt, J.-M., \& Sack, W. (2005). Thematic coherence and quotation practices in OSS design-oriented online discussions. In Proceedings of the 2005 International ACM SIGGROUP Conference on Supporting Group Work (GROUP '05) (pp. 177-186). Sanibel Island, Florida, USA: ACM Press.

Berkowitz, M. W., \& Gibbs, J. C. (1979). A Preliminary Manual for Coding Transactive Features of Dyadic Discussion.

Bronckart, J. P. (1995). Theories of action, speech, natural language, and discourse. In J. V. Wertsch, P. D. Rio \& A. Alvarez (Eds.), Sociocultural Studies of Mind (pp. 75-91). New York: Cambridge University Press.

Cai, D., Shao, Z., He, X., Yan, X., \& Han, J. (2005). Mining hidden community in heterogeneous social networks. In Proceedings of the 3rd international workshop on link discovery (pp. 58-65). New York: ACM Press.

Castells, M. (2001). The Internet Galaxy: Reflections on the Internet, Business, and Society: Oxford University Press.

Cohen, A. P. (1985). The symbolic construction of community: Tavistock Publications.

Dillenbourg, P. (1999). What do you mean by "collaborative learning"? In P. Dillenbourg (Ed.), Collaborative Learning: Cognitive and Computational Approaches (pp. 1-19). Amsterdam: Elsevier.

Donath, J. S. (1998). Identity and deception in the virtual community. In P. Kollock \& M. Smith (Eds.), Communities in cyberspace. London: Routledge. 
Dwyer, N., \& Suthers, D. D. (2006). Consistent practices in artifact-mediated collaboration. International Journal of Computer-Supported Collaborative Learning, 1(4), 481-511.

Edwards, D. (1997). Discourse and Cognition. London: SAGE Publications.

Garfinkel, H. (1967). Studies in Ethnomethodology. Englewood Cliffs, New Jersey: Prentice-Hall.

Gibson, J. J. (1977). The theory of affordances. In R. Shaw \& J. Bransford (Eds.), Perceiving, Acting, and Knowing. Hilsdale, NJ: Lawrence Erlbaum Associates.

Hutchins, E. (1995). Cognition in the wild. Cambridge, MA: The MIT Press.

Jones, C., Dirckinck-Holmfeld, L., \& Lindstrom, B. (2006). A relational, indirect, meso-level approach to CSCL design in the next decade. Computer-Supported Collaborative Learning, 1(1), 35-56.

Joseph, S., Lid, V., \& Suthers, D. D. (2007). Transcendent Communities. In C. Chinn, G. Erkens \& S. Puntambekar (Eds.), The Computer Supported Collaborative Learning (CSCL) Conference 2007 (pp. 317-319). New Brunswick: International Society of the Learning Sciences.

Kaptelinin, V., \& Nardi, B. A. (2006). Acting with technology: Activity theory and interaction design. Cambridge: MIT Press.

Koschmann, T. (2002, January 7-11). Dewey's contribution to the foundations of CSCL research. Paper presented at the Proc. Computer Supported Collaborative Learning 2002, Boulder.

Koschmann, T., Stahl, G., \& Zemel, A. (2007). The Video Analyst's Manifesto (or The Implications of Garfinkel's Policies for Studying Practice within Design-Based Research). In R. Goldman, R. Pea, B. Barron \& S. J. Derry (Eds.), Video Research in the Learning Sciences. Mahwah, NJ: Lawrence Erlbaum Associates, Inc.

Koschmann, T., Zemel, A., Conlee-Stevens, M., Young, N., Robbs, J., \& Barnhart, A. (2005). How do people learn: Member's methods and communicative mediation. In R. Bromme, F. W. Hesse \& H. Spada (Eds.), Barriers and Biases in Computer-Mediated Knowledge Communication (and how they may be overcome) (pp. 265-294). Amsterdam: Kluwer Academic Press.

Latour, B. (2005a). Reassembing the social: An introduction to actor-network theory. New York: Oxford University Press.

Latour, B. (2005b). Reassembing the Social: An Introduction to Actor-Network-Theory. New York: Oxford University Press.

Medina, R., \& Suthers, D. D. (2008, June 24-28, 2008). Bringing Representational Practice From Log to Light. Paper presented at the International Conference for the Learning Sciences, Utrecht.

Norman, D. A. (1999, May-June). Affordance, Conventions, and Design. ACM Interactions, 6, 38-42.

Renninger, K., \& Shumar, W. (2002). Building Virtual Communities. Cambridge: Cambridge University Press.

Sacks, H., Schegloff, E. A., \& Jefferson, G. (1974). A simplest systematics for the organization of turntaking for conversation. Language, 50(4), 696-735.

Shipman, F. M., III, \& McCall, R. (1994). Supporting knowledge-base evolution with incremental formalization, CHI94 (pp. 285-291). Boston, MA: ACM Press.

Shipman III, F. M., \& McCall, R. (1994). Supporting knowledge-base evolution with incremental formalization. In Proceedings of the SIGCHI conference on Human factors in computing systems: Celebrating interdependence (pp. 285-291). Boston, MA: ACM Press.

Stahl, G. (2006). Group Cognition: Computer Support for Collaborative Knowledge Building. Cambridge, MA: MIT Press.

Star, S. L. (1990). The structure of ill-structured solutions: Boundary objects and heterogeneous distributed problem solving. In L. Gasser \& M. N. Huhns (Eds.), Distributed artificial intelligence (Vol. 2, pp. 37-54). San Francisco: Morgan Kaufmann.

Suthers, D. D. (2006a). A qualitative analysis of collaborative knowledge construction through shared representations Research and Practice in Technology Enhanced Learning, 1(2), 1-28.

Suthers, D. D. (2006b). Technology affordances for intersubjective meaning-making: A research agenda for CSCL. International Journal of Computers Supported Collaborative Learning, 1(3), 315-337. 
Suthers, D. D., Dwyer, N., Medina, R., \& Vatrapu, R. (2007a). A framework for analyzing interactional processes in online learning Annual Meeting of the American Educational Research Association (AERA). Chicago.

Suthers, D. D., Dwyer, N., Medina, R., \& Vatrapu, R. (2007b). A framework for eclectic analysis of collaborative interaction. In C. Chinn, G. Erkens \& S. Puntambekar (Eds.), The Computer Supported Collaborative Learning (CSCL) Conference 2007 (pp. 694-703). New Brunswick, NJ: International Society of the Learning Sciences.

Suthers, D. D., Dwyer, N., Vatrapu, R., \& Medina, R. (2007). An abstract transcript notation for analyzing interactional construction of meaning in online learning. In Proceedings of the 40th Hawai i International Conference on the System Sciences (HICSS-34), (CD-ROM). New Brunswick, NJ: Institute of Electrical and Electronics Engineers, Inc. (IEEE).

Suthers, D. D., Harada, V. H., Doane, W. E. J., Yukawa, J., Harris, B., \& Lid, V. (2004). Technologysupported systemic reform: An initial evaluation and reassessment. Paper presented at the Proceedings of the Sixth International Conference of the Learning Sciences, Santa Monica, CA, pp 537-544.

Suthers, D. D., \& Hundhausen, C. (2003). An experimental study of the effects of representational guidance on collaborative learning. Journal of the Learning Sciences, 12(2), 183-219.

Suthers, D. D., Hundhausen, C. D., \& Girardeau, L. E. (2003). Comparing the roles of representations in face-to-face and online computer supported collaborative learning. Computers \& Education, 41, 335 351.

Suthers, D. D., Vatrapu, R., Medina, R., Joseph, S., \& Dwyer, N. (in press). Beyond threaded discussion: Representational guidance in asynchronous collaborative learning environments. Computers \& Education, doi:10.1016/j.compedu.2006.10.007.

Suthers, D. D., Yukawa, J., \& Harada, V. H. (2007). An activity system analysis of a tripartite technology-supported partnership for school reform. Research and Practice in Technology Enhanced Learning, 2(2), 1-29.

Tajfel, H., \& Turner, J. C. (1986). The social identity theory of inter-group behavior. In S. Worchel \& L. W. Austin (Eds.), Psychology of intergroup relations. Chicago: Nelson-Hall.

Teasley, S. D., \& Roschelle, J. (1993). Constructing a joint problem space: The computer as a tool for sharing knowledge. In S. P. Lajoie \& S. J. Derry (Eds.), Computers as Cognitive Tools (pp. 229-258). Hillsdale, NJ: Lawrence Erlbaum Associates. 